\title{
Chondroitinase ABC Combined with Neurotrophin NT-3 Secretion and NR2D Expression Promotes Axonal Plasticity and Functional Recovery in Rats with Lateral Hemisection of the Spinal Cord
}

\author{
Guillermo García-Alías, ${ }^{1}$ Hayk A. Petrosyan, ${ }^{2,3}$ Lisa Schnell, ${ }^{4}$ Philip J. Horner, ${ }^{5}$ William J. Bowers, ${ }^{6}$ Lorne M. Mendell, ${ }^{3}$ \\ James W. Fawcett, ${ }^{1}$ and Victor L. Arvanian ${ }^{2,3}$ \\ ${ }^{1}$ Centre for Brain Repair, University of Cambridge, Cambridge CB2 0PY, United Kingdom, ${ }^{2}$ Northport Veterans Affairs Medical Center, Northport, New \\ York 11768, ${ }^{3}$ Department of Neurobiology and Behavior, State University of New York at Stony Brook, Stony Brook, New York 11794, ${ }^{4}$ University of Zurich \\ and ETH Zurich, Brain Research Institute, CH-8057 Zurich, Switzerland, ${ }^{5}$ University of Washington, Institute for Stem Cell and Regenerative Medicine, \\ Seattle, Washington 98195, and ' Department of Neurology, Center for Neural Development and Disease, University of Rochester Medical Center, Rochester, \\ New York 14642
}

Elevating spinal levels of neurotrophin NT-3 (NT3) while increasing expression of the NR2D subunit of the NMDA receptor using a HSV viral construct promotes formation of novel multisynaptic projections from lateral white matter (LWM) axons to motoneurons in neonates. However, this treatment is ineffective after postnatal day 10. Because chondroitinase $A B C(C h A B C)$ treatment restores plasticity in the adult CNS, we have added ChABC to this treatment and applied the combination to adult rats receiving a left lateral hemisection $(\mathrm{Hx})$ at T8. All hemisected animals initially dragged the ipsilateral hindpaw and displayed abnormal gait. Rats treated with ChABC or NT3/HSV-NR2D recovered partial hindlimb locomotor function, but animals receiving combined therapy displayed the most improved body stability and interlimb coordination [Basso-Beattie-Bresnahan (BBB) locomotor scale and gait analysis]. Electrical stimulation of the left LWM at T6 did not evoke any synaptic response in ipsilateral L5 motoneurons of control hemisected animals, indicating interruption of the white matter. Only animals with the full combination treatment recovered consistent multisynaptic responses in these motoneurons indicating formation of a detour pathway around the $\mathrm{Hx}$. These physiological findings were supported by the observation of increased branching of both cut and intact LWM axons into the gray matter near the injury. ChABC-treated animals displayed more sprouting than control animals and those receiving NT3/HSV-NR2D; animals receiving the combination of all three treatments showed the most sprouting. Our results indicate that therapies aimed at increasing plasticity, promoting axon growth and modulating synaptic function have synergistic effects and promote better functional recovery than if applied individually.

\section{Introduction}

Damaged axons in the spinal cord do not regenerate spontaneously. This is due to the low intrinsic capacity of axotomized neurons to grow their axons (Hill et al., 2001) and the formation

\footnotetext{
Received Aug. 22, 2011; revised 0ct. 11, 2011; accepted 0ct. 14, 2011

Author contributions: G.G.-A., L.S., L.M.M., J.W.F., and V.L.A. designed research; G.G.-A., H.A.P., L.S., and V.L.A. performed research; P.J.H. and W.J.B. contributed unpublished reagents/analytic tools; G.G.-A., H.A.P., L.S., P.J.H., W.J.B., L.M.M., J.W.F., and V.L.A. analyzed data; G.G.-A., L.M.M., J.W.F., and V.L.A. wrote the paper.

The research was supported by Merit Review Funding from the Department of Veterans Affairs (V.L.A.), the Christopher and Dana Reeve Foundation (L.M.M., J.W.F.), the New York State Spinal Cord Injury Research Board (V.L.A.), the Department of Defense (V.L.A.), NIH Grant 5 R01 NS 16996 (L.M.M.), and the William J. Heiser Foundation (LMM). We thank Dr. Li Lou (State University of New York at Stony Brook) and Valentina Alessi (Northport Veterans Affairs Medical (enter) for help with immunochemistry experiments.

The authors declare no competing financial interests.

Correspondence should be addressed to Dr. Victor L. Arvanian, Northport Veterans Affairs Medical Center, 79 Middleville Road, Building 62, Northport, NY 11768. E-mail: vlarvanian@notes.cc.sunysb.edu.

G. García-Alías' present address: Department of Integrative Biology \& Physiology, University of California, Los Angeles, Los Angeles, CA 90095.

DOI:10.1523/JNEUROSCI.4308-11.2011

Copyright $\odot 2011$ the authors $\quad 0270-6474 / 11 / 3117788-12 \$ 15.00 / 0$
}

of an inhibitory environment, including perineuronal nets around neurons and their dendrites, which restricts regenerative axon growth and the formation of new connections (Snow et al., 1990; Silver and Miller, 2004; Busch and Silver, 2007; Galtrey et al., 2007; Carulli et al., 2010).

There are several experimental therapies that partially overcome these limitations and promote functional recovery in different animal models. Among these therapies, blockade of inhibitory NogoA (Maier and Schwab, 2006), digestion of chondroitin sulfate proteoglycans (CSPGs) (Kwok et al., 2008), neurotrophin delivery (Lu and Tuszynski, 2008), intervention in axon growth signaling pathways (Shearer et al., 2003) and physical rehabilitation (Girgis et al., 2007), have been shown to promote regeneration and/or sprouting of damaged and undamaged axons leading to functional repair. However, none of these interventions by itself gives full recovery of function. There is therefore a need to rationally combine treatments that address the multifactorial cellular and molecular events which impede spinal cord repair. Successful repair will probably require the integra- 

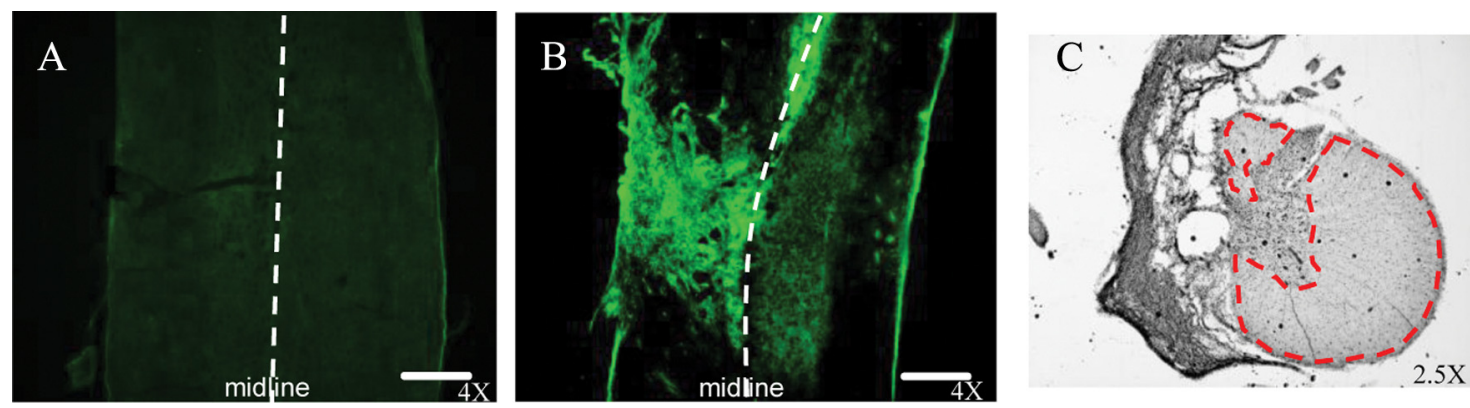

Figure 1. Spinal cord injury and accumulation of CSPGs at the injury epicenter after chronic Hx. A, Horizontal section of the cord prepared immediately after Hx and immunolabeled with CS-56 showing a low level of CSPGS. B, Horizontal section of the cord 3 weeks after Hx and immunolabeled with CS-56 showing accumulation of CSPGs in the vicinity of the lesion and in the contralateral white matter. C, Example of transverse section at SCl epicenter to show extent of Hx injury; prepared from the spinal cord whose electrophysiological responses are presented in Figure 5 ; ; highlighted is area of spared white matter. Scale bars, $100 \mu \mathrm{m}$.

tion of more than one treatment, and digestion of CSPGs has been successfully combined with rehabilitation, bridge grafts, and neurotrophin NT-3 (NT3), stem cell, and glial cell transplantation (Fouad et al., 2005; Houle et al., 2006; Massey et al., 2008; García-Alías et al., 2009; Karimi-Abdolrezaee et al., 2010; Alilain et al., 2011).

In the present study, we have combined two previous approaches, focused on the function of descending tracts in the lateral white matter (LWM). These connections from LWM, including ventrolateral funiculus (VLF), are of particular interest because of their role in the control of locomotion (Schucht et al., 2002; Alstermark et al., 2004; Reed et al., 2008). Previous studies in newborn animals have shown that the strength of the excitatory synaptic connection from VLF axons to lumbar motoneurons can be enhanced by NT3 (Arvanian et al., 2003), but novel polysynaptic connections required to provide detours around lesions are not (Arvanian et al., 2006). Enhancing expression of the NR2D subunit of NMDA receptor, normally downregulated in neonatal rats (Arvanian et al., 2004), promotes the appearance of novel polysynaptic connections in neonates (Arvanian et al., 2006).

Since synaptic plasticity is diminished by CSPGs in the extracellular matrix in the adult spinal cord (Kwok et al., 2008), we have added a third treatment, the enzyme chondroitinase ABC (ChABC), to the combined neurotrophin and NR2D intervention. ChABC digests the glycosaminoglycan chains of CSGPs and restores plasticity to the adult spinal cord and brain, mainly by digestion of CSPGs in perineuronal nets (Silver and Miller, 2004; Afshari et al., 2009).

To determine the effects of this combined approach, we used a lateral hemisection $(\mathrm{Hx})$ injury model, in which we could measure hindlimb motor function and study connectivity by electrophysiological recording and tracing axonal projections.

Some of these findings have been reported in abstract form (García-Alías et al., 2006; Lou et al., 2007).

\section{Materials and Methods}

All procedures were performed on adult female Sprague Dawley rats ( $\sim 200 \mathrm{~g})$ in compliance with the UK Animals (Scientific Procedures) Act 1986 and institutional guidelines, and by the Institutional Animal Care and Use Committee at State University of New York-Stony Brook and Northport Veterans Affairs Medical Center (VAMC). Because of the large number of animals needed to investigate the effects of the numerous treatments, three series of experiments were performed by the same investigators using identical procedures in the different laboratories. The experiments using electrophysiological analysis were performed at Stony Brook and Northport VAMC and those using axon tracing were done largely in Cambridge. Behavioral experiments were performed at both sites.

\section{Preparation of collagen plugs containing NT3-secreting or}

$\beta$-galactosidase-secreting fibroblasts

Cultured rat fibroblast cells genetically engineered to produce NT3 or $\beta$-galactosidase were suspended in $0.6 \%$ glucose-PBS to a final concentration of $0.4 \times 10^{6} \mathrm{cells} / \mu \mathrm{l}$, and a volume of $\sim 2 \mu \mathrm{l}$ was inserted in collagen plugs, as described previously (Kawaja and Gage, 1992; McTigue et al., 1998; Arvanian et al., 2006).

\section{HSV amplicon vector construction and packaging}

Packaging of helper virus-free amplicon vector stocks and subsequent virus purification and determination of amplicon virus titers via both expression- and transduction-based methodologies were performed as previously described (Bowers et al., 2000, 2001). Vectors carried genes for either NR2D (HSV-NR2D) or $\beta$-galactosidase (HSVlac), as well as the reporter gene green fluorescent protein $(G F P)$.

\section{Immunolabeling with CS-56 to determine the elevated level of}

CSPGs after chronic hemisection

The level of CSPGs was determined in horizontal sections using CS-56 immunolabeling (Fig. 1). Although it is not clear which exact glycosaminoglycans of CSPGs core proteins are recognized by the CS-56 antibody, it has been widely used as a marker for the injury-induced overexpression of CSPGs that are subject to ChABC digestion (Davies et al., 2006; Kim et al., 2006). The procedure for CS-56 immunolabeling has been previously described (Fidler et al., 1999). Briefly, all sections were circled with a hydrophobic resin and incubated for $1 \mathrm{~h}$ in a humidity chamber in PBS containing $0.4 \%$ Triton X-100 with 1:30 goat serum to block nonspecific binding. The sections were then kept in 1:400 mouse monoclonal antiCS-56 IgM (Sigma) overnight, rinsed in $0.1 \mathrm{~m}$ PBS containing $0.4 \%$ Triton X-100 with 1:100 goat serum three times and then incubated with 1:100 Alexa Fluor 488 goat-anti-mouse IgM (Invitrogen) for $3 \mathrm{~h}$. Preabsorption of the secondary antibody with rat serum was performed $1 \mathrm{~h}$ before use. After three rinses, the sections were coverslipped with Prolong Gold antifade reagent (Invitrogen) and viewed using a Zeiss Axioskop upright microscope with appropriate fluorescence bandpass filter and captured using a Spot RT camera with ImagePro Plus software (Media Cybernetics). All images were captured within $48 \mathrm{~h}$ of completing the staining procedures.

\section{Spinal cord injury and treatment delivery}

The animals were deeply anesthetized with a mixture of isoflurane (1.5 $\mathrm{L} / \mathrm{min})$ and $\mathrm{O}_{2}(0.4 \mathrm{~L} / \mathrm{min})$ and placed prone on a heating pad. The T8 vertebra was removed to expose the dorsal surface of the spinal cord. The meningeal layer was cut longitudinally, and the left hemicord was completely transected with the tip of an iridectomy scissors (Fig. 1C). Immediately after the injury, the animals received the following treatments.

Chondroitinase $A B C$ or pencillinase. Two injections ( $1 \mu \mathrm{l}$ each) of chondroitinase ABC (100 U/ml; Seikagaku Corporation) or its control 
A

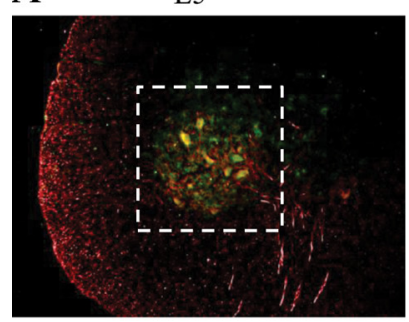

B

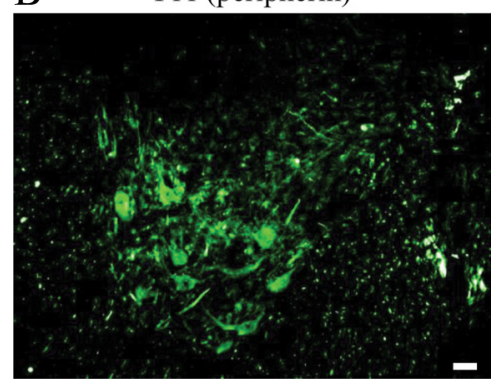

L5 (peripherin)

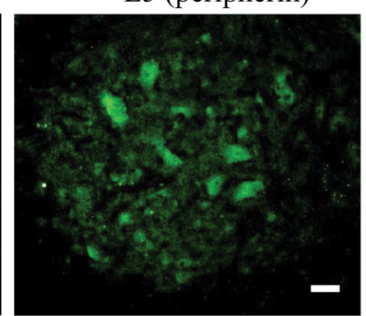

$\mathrm{T} 11$ (gfp)

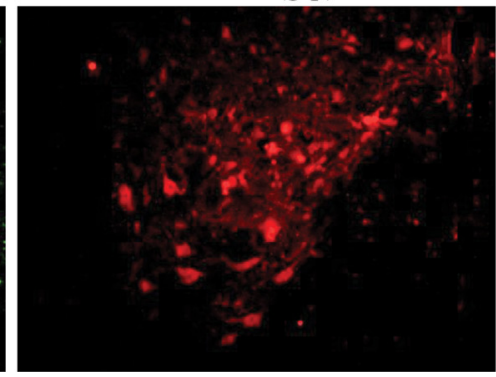

L5 (gfp)

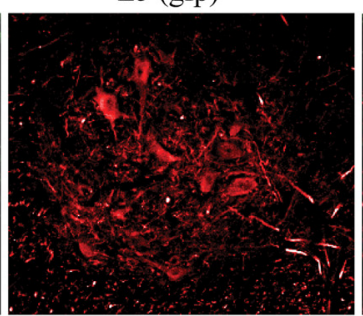

$1 /$

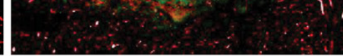

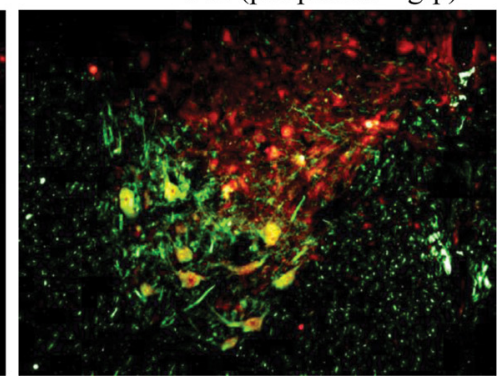

Figure 2. Transverse sections at L5 and T11 of an animal treated with NT3 + NR2D-gfp, double-stained for GFP (red, marker for GFP) and peripherin (green, marker for motoneurons), showing the expression NR2D in motoneurons and other cell populations. Scale bars: $A, B, 30 \mu \mathrm{m}$; lower-power image in $\boldsymbol{A}$ to show location of immunolabeled cells in ventral horn.

penicillinase ( $2 \mathrm{mg} / \mathrm{ml}$, Sigma) were made $1 \mathrm{~mm}$ rostral and caudal to the injury on the ipsilateral side near the midline. The injections were done over a period of $10 \mathrm{~min}$ using a glass micropipette (30 $\mu \mathrm{m}$ diameter) attached to a Hamilton syringe. This procedure was previously shown to reduce conduction block in fibers contralateral to chronic $\mathrm{Hx}$ (Hunanyan et al., 2010). Our earlier studies demonstrated CSPG digestion after similar chondroitinase injection, which was assessed immunohistochemically using the 1-B-5 antibody (which recognizes the sugar stub remaining on CSPG molecules after digestion of the glycosaminoglycan chains) (described in detail in our previous studies: García-Alías et al., 2008, 2009).

NR2D (HSV-NR2D) or $\beta$-galactosidase (HSVlac) vectors. HSV-1-gfp amplicons encoding NR2D or control $\beta$-galactosidase, were injected intraspinally ( 2 injections of $1 \mu \mathrm{l} \mathrm{each;} \sim 10^{4}$ viral particles/ $\mu \mathrm{l}$ ) into the left and right ventral horn just caudal to the injury region. HSV-1 amplicons have a transgene capacity sufficient to carry the NR2D cDNA and the coexpressed GFP reporter gene (Arvanian et al., 2004). HSV-1-gfpmediated gene expression peaks during the first $24-48 \mathrm{~h}$ (Bowers et al., $2000,2001)$. The motoneuron infection was determined by using double immunolabeling with peripherin (motoneurons marker, green) and with GFP (red). The immunolabeling procedure and analyses have previously been described (Arvanian et al., 2004). We found a substantial level of infection of motoneurons in the thoracic cord and even at a remote lumbar level, measured $7 \mathrm{~d}$ after injection of HSV-NR2D-gfp. Transverse spinal cord sections from three animals with HSV-NR2D-gfp injections showed immunofluorescence in spinal cord cells at the T11 and L5 spinal segments. Staining was particularly marked in large neurons in the ventral horn with the morphology of motoneurons, many of which were also positive for Peripherin (motoneuron marker) (Fig. 2). However, some cells were labeled with GFP (NR2D) but not peripherin, particularly at T11 (Fig. 2 B). Considering the size of these cells and the natural tropism of HSV1 for neurons (Lilley et al., 2001), these cells were likely interneurons. Consistent with these results, long-distance retrograde and anterograde transport of HSV-1 has been reported (Zemanick et al., 1991).

NT3-secreting or $\beta$-galactosidase-secreting fibroblast plugs. Collagen plugs shaped as a half cylinder $1 \mathrm{~mm}$ in diameter and $1.5 \mathrm{~mm}$ long, containing either NT-3-secreting or $\beta$-galactosidase-secreting fibroblasts $\left(0.4 \times 10^{6}\right.$ cells $/ \mu \mathrm{l}$, suspended in $0.6 \%$ glucose-PBS, cell volume of $2 \mu \mathrm{l}$ ) were placed flat side down directly on top of the cord at the level of injury as previously described (Kawaja and Gage, 1992; McTigue et al., 1998; Arvanian et al., 2003). These earlier studies demonstrate elevation of neurotrophin levels in the spinal cord and biological effects specific to the released neurotrophin days to weeks later following this procedure.

\section{Experimental groups}

Behavioral, electrophysiological and tracing experiments were performed on 4 groups of hemisected animals, each treated with different combinations of agents and/or controls for the agents. One group was treated with penicillinase (control for ChABC) + HSV-1ac vectors (control for NR2D expression) $+\beta$-galactosidase-secreting fibroblasts (control for NT-3) plugs (Group PEN, $n=24$ before exclusion). A second group was treated with penicillinase + HSV-NR2D vectors + NT-3secreting fibroblasts plugs (Group NT3/NR2D, $n=14$ before exclusion). A third group was treated with chondroitinase $\mathrm{ABC}+\mathrm{HSV}-1 \mathrm{ac}$ vectors + $\beta$-galactosidase-secreting fibroblasts plugs (Group ChABC, $n=22$ before exclusion). A fourth group was treated with chondroitinase $\mathrm{ABC}+$ HSV-NR2D vectors + NT-3-secreting fibroblasts plugs (Group COMB, $n=22$ before exclusion). All animals passing the initial exclusion test were maintained for 6-8 weeks after $\mathrm{Hx}$ and treatment, after which electrophysiology or tracing studies were carried out. During the 6- to 8 -week postsurgery period, some but not all animals in each group were tested behaviorally for locomotor and sensory function (see below). The number of rats in each treatment group subjected to behavioral, electrophysiological or tracing studies is given in conjunction with the description of their results.

An additional control group that received no injury and control treatment with penicillinase $+\mathrm{HSV}$-1ac vectors $+\beta$-galactosidase-secreting fibroblasts plugs ( $n=6$ ) (Group Noninjured control) was used for electrophysiology only. Additional animals that received hemisection injury and were perfused $20 \min (n=3)$ or 3 weeks $(n=3)$ after the injury were used to determine the level of CSPG expression in the vicinity of the injury. An additional 3 animals that received $\mathrm{Hx}$ injury and intraspinal injections of HSV1-gfp-NR2D were used to confirm the ability of HSV-1 to infect cells at thoracic and lumbar levels.

\section{Exclusion of animals}

Preservation of only $5 \%$ of the ventral white matter on the lesioned side has been shown to be enough for the animals to rapidly recover hindlimb locomotion (You et al., 2003). Therefore, to exclude animals with probable ventral tissue preservation, animals that could use their left hindlimb at 1 day postoperation (dpo) were eliminated from further consideration. In a few cases animals displayed a less than complete hemisection upon postmortem histological examination were also excluded from the final dataset.

\section{Locomotor evaluation}

Locomotor tests were performed before the operation and at 4, 7, 14, 21, 35, and 42 dpo. Hindlimb locomotor behavior was assessed with the 
Basso-Beattie-Bresnahan (BBB) scale. Animals were placed individually in the middle of a circular enclosure and allowed to move freely for $5 \mathrm{~min}$. Two observers evaluated and scored hindlimb performance according to the BBB scale, ranging from 0 (no movement) to 21 (normal movement) (Basso et al., 1995). Body weight support and limb coordination were assessed by foot print analysis. The fore- and hindpaws of the animals were inked with different colors and footprints were made on paper covering a $8 \mathrm{~cm}$ narrow, $100 \mathrm{~cm}$ long runway as the animals walked across. Hindlimb footprints were used to determine the animal's body weight support by calculating the mean stride length, base of support and the hindlimb rotation angle. The stride length was measured between the central pads of two consecutive prints on each side, the base of support was measured as the core-to-core distance of the print representing the central pad, and the rotation angle was measured as the angle formed by the intersection of the line which joins the print of the third digit and the central pad and the line through the metatarsophalangeal print parallel to the walking direction. To assess limb coordination, the number of foreand hindpaws prints was counted and the distance between the central pads of the fore- and hindlimb on each side of the body was measured (Metz and Schwab, 2004).

To evaluate the hindlimb's capacity for skilled movement, we observed the animals while crossing a 1-meter horizontal ladder with irregularly placed bars and counted the number of the left and right hindlimb's foot slips (errors) (Liebscher et al., 2005). These measurements were made before the $\mathrm{Hx}$ and at 7 and $42 \mathrm{dpo}$.

\section{Sensory evaluation}

Thermal and mechanical sensibilities were tested at the end of the functional evaluation. To evaluate thermal hyperalgesia, the rats were placed individually inside a Plexiglas box elevated above the floor, and a radiant heat stimulus was focused onto the plantar surface of the hindpaw. The time to withdrawal the heated paw was measured automatically using an infrared detector directed to the plantar surface (Ugo Basile). The maximum time of stimulation was limited to $32 \mathrm{~s}$ to avoid skin damage. The response time was scored as the mean of three trials separated by 5 min resting periods (Hargreaves et al., 1988). Mechanical sensitization was measured using an electronic von Frey Anesthesiometer (Model 1601C, Life Science Instruments). The probe was applied to the glabrous skin of the plantar surface of the hindpaw, and the pressure was gradually increased until the rat withdrew its paw. The force transducer recorded the maximum force applied in grams. If the rat did not withdraw after $500 \mathrm{~g}$ had been applied, the test was terminated. This procedure was repeated for three trials on each paw. The trials were performed on the left and right hindpaws alternately (Galtrey et al., 2007).

\section{Electrophysiological evaluation}

After completing the behavioral evaluation, one subgroup of rats was studied electrophysiologically to determine whether novel functional projections had developed around the Hx. The rats were deeply anesthetized using a ketamine $(80 \mathrm{mg} / \mathrm{kg}, 0.5 \mathrm{ml}) /$ xylazine $(10 \mathrm{mg} / \mathrm{kg}, 0.5 \mathrm{ml})$ mixture intraperitoneally. Heart rate and expired $\mathrm{CO}_{2}$ were monitored continuously. Dorsal laminectomy of the spinal cord was performed with two openings, at thoracic T6-T8 (for placement of stimulation electrodes) and lumbar L1-L6 (for placement of recording electrodes at L5).

For recordings we used Axoprobe 1A amplifier (10,000 Hz sampling rate). Motoneurons were impaled with sharp glass microelectrodes ( $3 \mathrm{M}$ $\mathrm{K}$ acetate; $50-70 \mathrm{M} \Omega$ resistance) and identified by their antidromic response to stimulation of the cut L5 ipsilateral ventral root. The resting membrane potential of motoneurons used for analysis ranged from -45 to $-65 \mathrm{mV}$. For electrical stimulation we used a tungsten electrode insulated close to the tip and measuring $100 \mathrm{~K} \Omega$. This electrode was inserted first in the left and then in the right thoracic cord at T6 (rostral to the level of the $\mathrm{Hx}$ ) and was positioned between the dorsal root entry zone and lateral edge via a micromanipulator. It was lowered to a depth of $1.3 \mathrm{~mm}$ to recruit axons of rubrospinal and reticulospinal tracts as previously described (Hunanyan et al., 2011). The stimulus had duration of $50 \mu \mathrm{s}$ and was delivered at $0.1 \mathrm{or} 1 \mathrm{~Hz}$. The stimulus intensity required to evoke a maximum response usually ranged from 40 to $100 \mu \mathrm{A}$ in intact cords and from 300 to $600 \mu \mathrm{A}$ in chronically hemisected cords. The maximum synaptic response for each cell was determined by increasing the stimulus intensity until the response reached a plateau. EPSP amplitude was measured $(0-100 \%)$ and responses at or below baseline synaptic noise level $(\leq 0.2 \mathrm{mV})$ were considered "no response". Peak EPSP amplitude was measured from prestimulus baseline to peak. Latency was measured from stimulus artifact to response onset. We usually recorded from 5-6 motoneurons per rat, each one tested with lateral white matter stimulation on each side. After completion of electrophysiological recording, the rats were perfused, spinal cords were removed and the injury was verified by reconstruction (Arvanian et al., 2009).

\section{Anterograde axon tracing}

At the end of the behavioral evaluation, a second subgroup of rats in each treatment group received bilateral stereotaxic injections of $1 \mu \mathrm{l}$ of $10 \%$ biotinylated dextran amine (BDA) in the gigantocellular nucleus of the reticular formation [coordinates anteroposterior $-11, \mathrm{ML} \pm 1$, and 10 $\mathrm{mm}$ deep (Paxinos and Watson, 1998)] under sterile conditions (Reed et al., 2008). These injections label reticulospinal axons in the spinal cord. In another subgroup of animals, a wide laminectomy was performed at T6 and the surface of the dorsal and lateral funiculi was exposed. A longitudinal cut was made in the dura mater and the tip of a glass micropipette attached to a Hamilton syringe was introduced to a depth $1 \mathrm{~mm}$ in the right ventrolateral funiculus at T6 (corresponding to the position of stimulating electrode in the electrophysiological experiments), avoiding penetration of the dorsal roots. Under manual pressure $1 \mu \mathrm{l}$ of the water-soluble tracer dextran conjugated to Alexa Fluor 594 (Invitrogen) was injected over a period of $10 \mathrm{~min}$. This procedure was repeated to inject dextran in the left VLF at T6. Finally, the muscles and skin were sutured in layers, and the skin disinfected. These injections label axons in the ventrolateral cord white matter, representing several tracts (see below).

Two weeks (BDA tracing) or 4 weeks later (dextran), the animals were deeply anesthetized and intracardially perfused with $200 \mathrm{ml}$ of PBS $0.1 \mathrm{M}$, $\mathrm{pH}=7,4$, followed by $300 \mathrm{ml}$ of PFA $4 \%$ in the same solution. The spinal cords were dissected, and a block including the T6-T9 segment was removed and kept overnight in the same solution at $4^{\circ} \mathrm{C}$. The following day, the spinal blocks were cryoprotected in $30 \%$ sucrose and stored until further analysis. Longitudinal sections, $40 \mu \mathrm{m}$ thick, were cut and alternately processed for reticulospinal axon visualization [the avidin-biotin amplification method with peroxide as a substrate (Vectastain ABC Elite Kit, Vector Labs)] and stained with diaminobenzidine and $\mathrm{NiCl}_{2}$, or stained with cresyl violet for measuring the extent of the injury, or examined under the fluorescence microscope using filters appropriate for visualizing Alexa Fluor 594-labeled axons.

\section{Axon crossing quantification}

For the axons labeled by intraspinal injections we counted the number of processes in the right and left lateral funiculi crossing the boundary between the white and gray matter, in an area extending $2 \mathrm{~mm}$ rostral and $2 \mathrm{~mm}$ caudal to the injury. The number of crossing axons was normalized for each animal by dividing the number of axon crossings by the total number of fibers traced in the right and left lateral funiculi and the length of the section studied (Girgis et al., 2007; García-Alías et al., 2009). For the reticulospinal axons we counted processes crossing the gray-white matter boundary divided by the number of axons, as above. However, this count was performed separately for right and left sides of the cord, for the left side $2 \mathrm{~mm}$ above the lesion, and for the right side $2 \mathrm{~mm}$ above and $2 \mathrm{~mm}$ below the lesion. In addition we counted the total number of axon branches emanating from the labeled parent fibers in the white matter in the direction of the gray matter. We count both branching axons and those entering gray matter as measures of axon sprouting and of the number of connections that will be formed within the local gray matter.

\section{Statistical analysis}

Results are shown as the mean \pm SEM. For the electrophysiology the peak amplitude of EPSPs from all motoneurons (30 consecutive responses per cell) were averaged over all motoneurons recorded in each rat and compared using one-way ANOVA or one-way ANOVA on Ranks ( $n$ is number of rats). If significant differences were observed between groups, a Student-Newman-Keuls test or Dunn's method were used for pairwise comparisons (SigmaPlot 11.0 software). Behavioral and histological 
a Footprints analysis
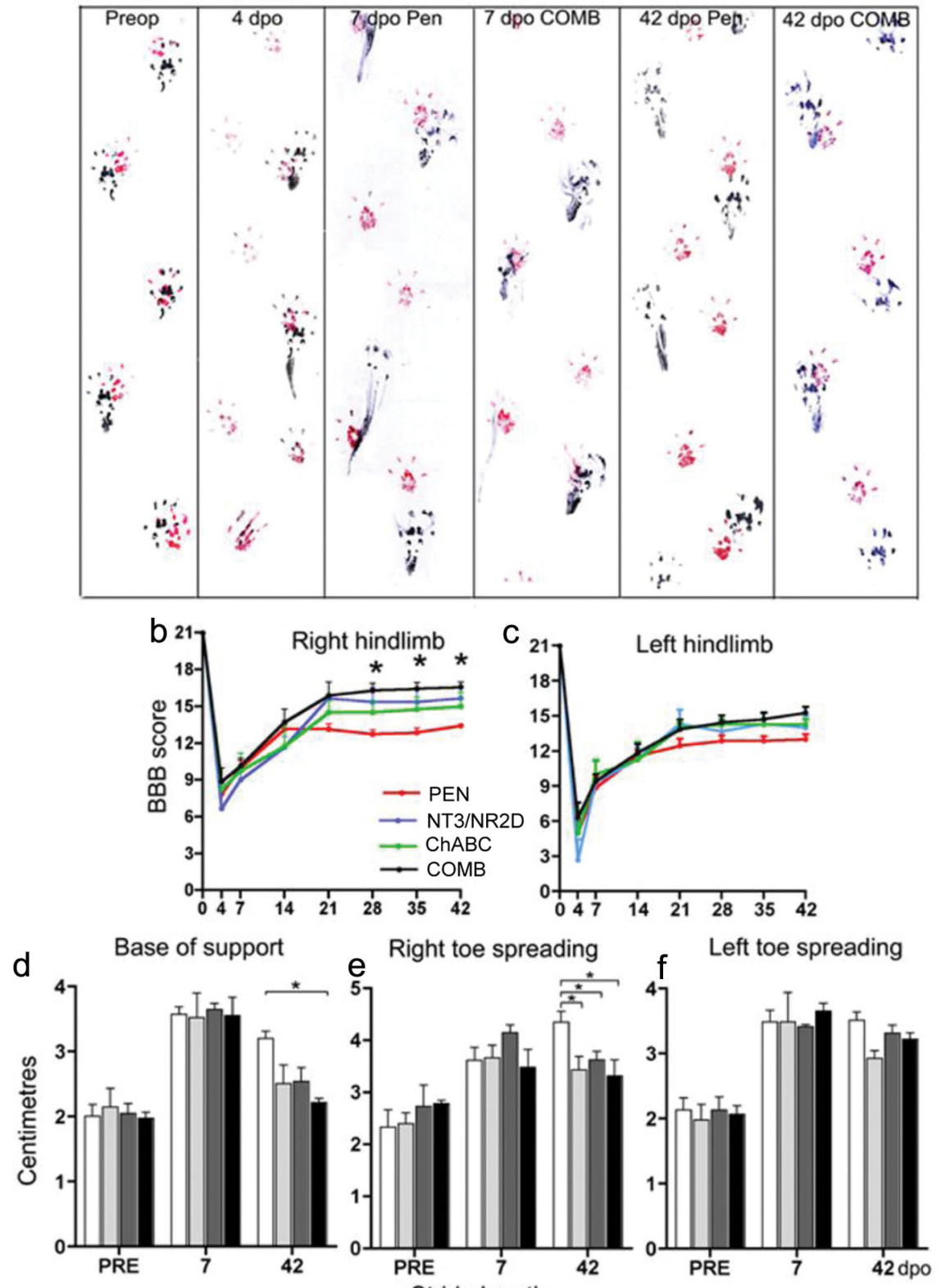

\section{g}
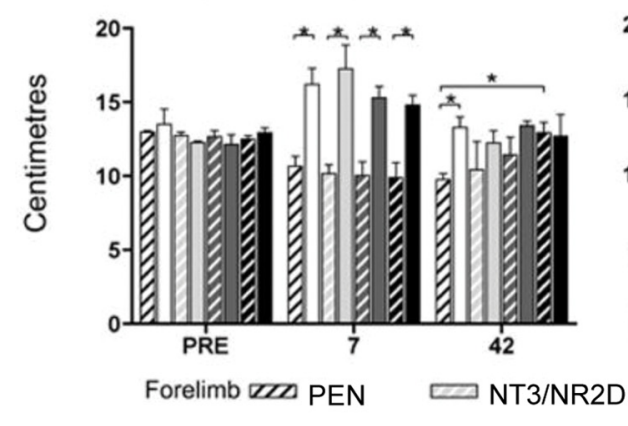

Hindlimb $\square$ PEN

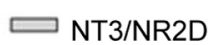

h

Left fore- and hind-limb

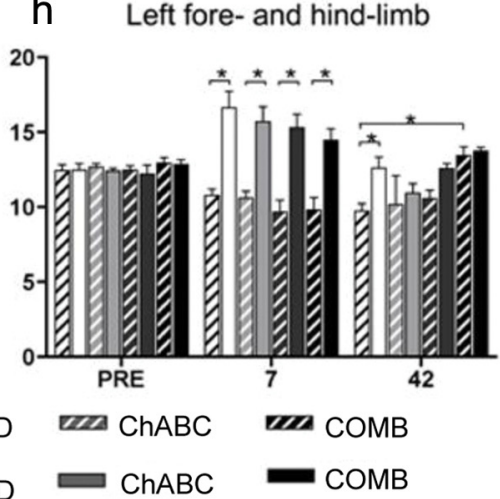

Figure 3. Locomotor recovery after injury and treatment. $\boldsymbol{a}$, Footprints of PEN and COMB animals before injury and at different time points postinjury illustrating the placement of the fore- and hindpaws when walking across a runaway. $\boldsymbol{b}, \boldsymbol{c}$, Following the injury, the animals had locomotor impairment of the right and left hindlimbs, as measured with the BBB scale, which partially recovered over time. At $42 \mathrm{dpo}$ the animals were able to support their weight, plantar step, and in addition COMB animals recovered interlimb coordination ( ${ }^{*} p<0.05$, compare COMB $(n=10)$ and PEN groups $(n=9)$. Analysis using the footprints complements the results obtained with the BBB testing. $\boldsymbol{d}-\boldsymbol{h}$, Base of support (d), right and left toe spreading $(\boldsymbol{e}, \boldsymbol{f})$, and right and results were analyzed with ANOVA, both followed with Bonferroni post hoc analysis. Results were considered to be significant for $p<0.05$.

\section{Results}

Spinal cord injury and increased level of CSPGs after chronic hemisection

Animals received a left hemisection at $\mathrm{T} 8$. Two months postinjury, histology at the level of the injury showed a cystic cavity which extended a few millimeters in the rostral-caudal axis, completely transecting the white matter and gray matter on the left side of the cord (Fig. 1). The width and length of the cystic cavity were measured in all animals, and no differences were found between groups.

The level of CSPGs in the vicinity of the hemisection was assessed immunohistochemically using the CS-56 antibody. In horizontal sections prepared $20 \mathrm{~min}$ after $\mathrm{Hx}(n=3)$, the level of CS-56 immunostaining was negligible (Fig. 1A). However, in sections prepared from the rats 3 weeks postoperation $(n=3)$, we observed a dense immunosignal in the vicinity of the lesion, extending $1-1.5 \mathrm{~mm}$ rostralcaudal along the central canal and into contralateral white matter (Fig. 1B).

\section{Effects on behavior}

LWM axons including those in the reticulospinal tract play an important part in the control of hindlimb locomotion (Schucht et al., 2002), and we therefore focused on recovery of hindlimb motor function. Lateral hemisection of the thoracic spinal cord leads to transient severe impairment of hindlimb function ipsilateral to the injury (Hains et al., 2001; Ballermann and Fouad, 2006; Arvanian et al., 2009) with milder effects on the contralateral hindlimb. All the animals tested showed a similar pattern of recovery in the 2 weeks after injury. The first day postinjury, the animals dragged the hindlimb ipsilateral to the injury, and during the following days recovered weight support and plantar placement of the paw when stepping. Other motor impairments of the left and right hindlimbs also recovered partially during the following weeks. As illustrated in Figure 3a, before the injury the animals

left fore- and hindlimb stride length $(\boldsymbol{g}, \boldsymbol{h}) \cdot \boldsymbol{d}$ shows that by day 42 , treated animals, particularly in the COMB group, have recovered to an almost normal base of support, indicating recovery of postural control. This is accompanied by a recovery in interlimb coordination, another indicator of motor control, with fore- and hindlimb stepping distances recovered to normal by day 42 in the COMB group [compare the two right bars (COMB) at day 42 in $\boldsymbol{g}$ and $\boldsymbol{h}$ with the two left bars (PEN) $\left.\left({ }^{*} p<0.05\right)\right]$. 
exhibited stable and coordinated stepping, with a narrow hindlimb base of support, partial toe spreading and with overlapping forepaw and hindpaw footprints on each side. Four days after injury, the animals in all the groups were able to support their weight with the right hindpaw (Fig. $3 a, 4$ dpo) but not with the left hindpaw which was dragged. No difference was found between groups at this time point. At 7 dpo the animals had regained partial left hindlimb body weight support, but locomotion was unstable, as evidenced by a wide hindlimb base of weight support (Fig. $3 d$ ), elevated right and left hindpaw toe spreading (Fig. $3 e, f$ ) and by lack of fore- and hindlimb coordination, as measured by the difference in stride length between forelimbs and hindlimbs (Fig. $3 g, h$ ). At 42 dpo control untreated PEN animals showed the same deficits as at $14 \mathrm{dpo}(n=9)$. In contrast, treated animals in all groups showed substantial improvements with most of the gait measures returning almost to normal. There was a nonsignificant trend with the COMB group $(n=10)$ achieving slightly better scores in most categories than the animals treated with individual agents (ChABC, $n=9$; NT-3/NR2D, $n=6)$. Relative to the control PEN animals the COMB-treated animals displayed narrower base of body support ( $p<0.05$ vs PEN), reduction in right toe spreading ( $p<0.05$ vs PEN), an increase in forelimb stride length to a value similar to that for the hindlimbs ( $p<0.05$ vs PEN) (Fig. $3 g, h$ ), and return of almost normal hindlimb-forelimb coordination (Fig. 3a, 42 dpo COMB; compare with the neighboring PEN recording). Both the decrease in the base of weight support, and the ability of the animals to coordinate forelimb and hindlimb stepping, achieved at $42 \mathrm{dpo}$ by the COMB group, indicate that animals had largely recovered their ability to control their posture, allowing them in turn to achieve an organized gait.

Before the injury, the animals were trained to walk across a ladder with irregularly placed rungs and made few missteps. Seven days after the injury all animals made more right and left hindlimb missteps, completely or partially missing the rung. At the end of the evaluation the animals in all groups had partially recovered with a reduced number of mistakes, particularly with the right hindlimb. However, there were no significant differences between the experimental groups.

At the end of the evaluation period we tested mechanical and thermal sensation. All the animals showed mechanical hypersensitivity of both right and left hindpaws, with a lower threshold for paw withdrawal compared with noninjured animals $(p<0.001)$. No differences were found between groups or between right and left hindpaws (Fig. 4a). The increased mechanical sensitivity was not sufficient to cause behavioral changes such as protective behavior. Thermal sensation showed a trend to mild hypersensitivity in the injured animals, but there were no significant differences between groups or between right and left hindpaws (Fig. 4b).

\section{Electrophysiological results}

A total of 30 rats were tested electrophysiologically 6-8 weeks after injury to study changes in the white matter projections. The results from six cords with incomplete $\mathrm{Hx}$ (1 from PEN group, 2 from ChABC group, 2 from NT3/NR2D group and 1 from COMB group) were excluded from the results leaving a total of 24 rats. The animals were deeply anesthetized and the spinal cords were exposed. L5 motoneurons were impaled with a sharp glass micropipette electrode and a tungsten sharp electrode was introduced at a depth of $1.3 \mathrm{~mm}$, first in the right and then in the left LWM at T6 to stimulate descending spinal axons, as in previous experiments (Arvanian et al., 2009; Hunanyan et al., 2010).
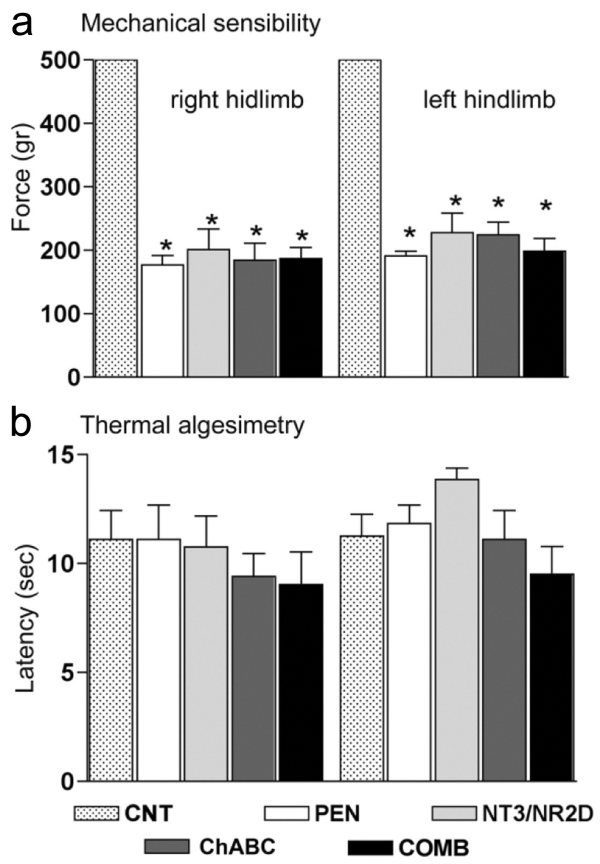

Figure 4. Thermal and mechanical sensitivity after spinal cord injury and treatment. $\boldsymbol{a}$, At $42 \mathrm{~d}$ postinjury the animals exhibited sensitization to mechanical stimuli $\left[{ }^{*} p<0.001\right.$ vs CNT (Control)]. No differences were found between treated groups. $\boldsymbol{b}$, None of the experimental groups exhibited thermal hyperalgesia.

In uninjured animals that received control treatments, single stimuli to the left lateral white matter at T6 gave rise to short latency EPSPs recorded in ipsilateral L5 motoneurons (Fig. 5a; amplitude $6.7 \pm 0.6 \mathrm{mV}$; latency $1.4 \pm 0.2 \mathrm{~ms}, n=6$ rats). Short latency, steep rising phase and negligible fluctuation in both latency and amplitude suggested that these responses were most probably monosynaptic, as previously discussed (Arvanian et al., 2009). Previous work indicates these connections are glutamatergic (Hunanyan et al., 2010, 2011). In the same animals, subsequent stimulation of the right lateral white matter at T6 gave rise to a long latency polyphasic EPSPs with a less steep rising phase in the same left L5 motoneurons, which were variable in shape and peak deflection (Fig. $5 a$; amplitude $2.9 \pm 0.4 \mathrm{mV}$; latency $4.9 \pm$ $0.5 \mathrm{~ms} ; n=6$ rats). These parameters indicate polysynaptic transmission from the right LWM to left L5 motoneurons (Arvanian et al., 2009).

Six to 8 weeks after injury PEN-treated animals exhibited no detectable EPSPs in L5 motoneurons in response to stimulation of the ipsilesional (left) LWM at level T6, even at high stimulation intensity (Fig. $5 b$ ). Stimulation of the contralateral (right) LWM at T6 evoked barely detectable EPSPs $(0.24 \pm 0.2 \mathrm{mV})$ in only 2 of 5 rats recorded. ChABC animals also displayed no EPSPs in L5 motoneurons after stimulation of the ipsilateral LWM (Fig. 5c), but there was a small EPSP present in L5 motoneurons after stimulation of the contralateral right LWM in 4 of 7 rats recorded (amplitude $0.82 \pm 0.35 \mathrm{mV}$; latency $5.4 \pm 0.5 \mathrm{~ms}$ ). The EPSPs observed in these crossed LWM-motoneuron projections exhibited a long latency, and a time course similar to that observed in control unlesioned animals (Fig. 5c). In a few NT3-treated animals with enhanced NR2D expression ( 3 of 6 rats recorded) we observed long latency, small amplitude EPSPs from both ipsilateral LWM (amplitude $0.65 \pm 0.22 \mathrm{mV}$; latency $5.8 \pm 0.7 \mathrm{~ms}$ ) and contralateral LWM (amplitude $0.78 \pm 0.3 \mathrm{mV}$, latency $5.3 \pm 0.6$ $\mathrm{ms})$. Animals treated with the COMB treatment of all three agents showed robust responses to stimulation of both left and 
right LWM in all 6 rats recorded (Fig. 5e). However, the shape of the responses evoked from the left T6 LWM to ipsilateral L5 motoneurons in injured and COMB-treated animals was strikingly different from the shape of monosynaptic responses in noninjured spinal cord (Fig. 5) in having a longer delay (latency $5.7 \pm 0.6$ $\mathrm{ms}$ ) and longer duration, reminiscent of the crossed polysynaptic EPSPs seen in uninjured animals.

The amplitude of EPSPs from ipsilateral (left) LWM in COMB animals $(1.62 \pm 0.38 \mathrm{mV})$ was greater than that in preparations treated with ChABC or NT3 and enhanced NR2D expression $(p<$ 0.05) (Fig. 5). It was also substantially larger than the linear sum of these responses suggesting a cooperative effect between these treatment modalities. Stimulation of the contralateral (right) LWM in COMB animals gave rise to delayed evoked potentials in the left L5 motoneurons, which had a similar time course but a greater amplitude (amplitude $1.95 \pm 0.42 \mathrm{mV}$, latency $5.3 \pm 0.6 \mathrm{~ms})$ than that seen in animals treated with ChABC $(p<0.05)$ or NT3 and enhanced NR2D expression $(p<0.05)$ alone. However, the amplitude of the response to contralateral LWM in COMB-treated rats was similar to that expected from summing the results from the individual treatments, i.e., no apparent cooperative effect (see Discussion).

We investigated whether the size of the responses is correlated with the area of spared white matter contralateral to $\mathrm{Hx}$. The injury was reconstructed from consecutive sections in the vicinity of $\mathrm{Hx}$, and the area of spared lateral and dorsal white matter was measured (the measured area is identified in Fig. 1C). There was no gross difference in the measured area (in $\mathrm{mm}^{2}$ ) of the spared contralateral white matter (dorsal column/ lateral column) at the injury epicenter in the different treatment groups ( $n=$ number of animals): PEN: $0.23 \pm 0.04 / 1.32 \pm 0.04$ $(n=5)$; ChABC: $0.21 \pm 0.03 / 1.21 \pm 0.03(n=7)$; NT3/NR2D: $0.21 \pm 0.02 / 1.38 \pm 0.05(n=6)$; COMB: $0.20 \pm 0.03 / 1.25 \pm 0.06$ $(n=6)$. Calculation of the Pearson coefficient revealed no significant correlation between EPSPs peak amplitude and area of spared white matter at injury epicenter: $\left(r^{2}=0.35, p=0.45\right.$ PEN $),\left(r^{2}=0.25, p=0.31\right.$ ChABC $),\left(r^{2}=0.28, p=0.53 \mathrm{NT} 3 /\right.$ NR2D $),\left(r^{2}=0.21, p=0.61\right.$ COMB $)$. Thus the amplitude of the responses was determined rather by the treatment that the rats received.

\section{Spinal axon morphology and sprouting}

BDA was applied bilaterally to the brainstem reticular formation to study the effects of the lesion and the different treatments on sprouting of reticulospinal axons. These axons innervate spinal neurons predominantly in the cervical and lumbar enlargements. Along their pathway some reticulospinal axons decussate (Reed
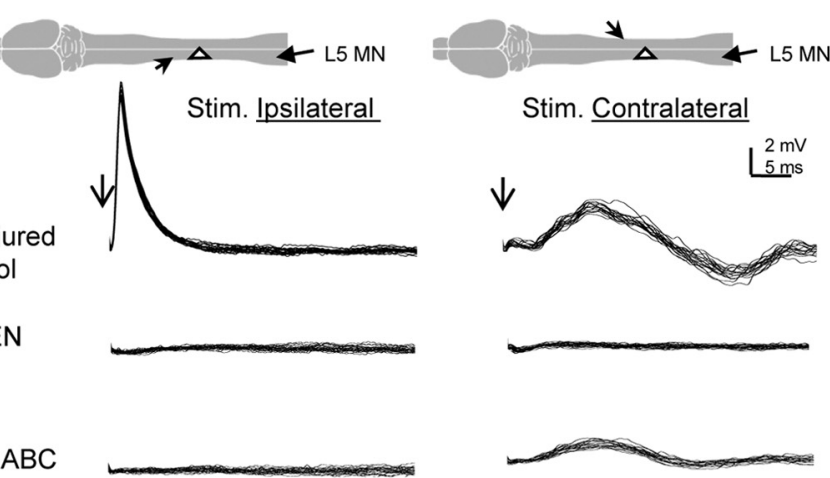

d HX, NT3/NR2D
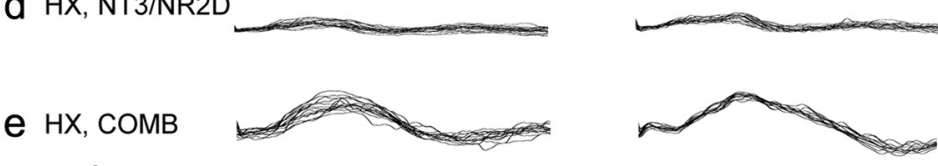

Figure 5. LWM stimulation and intracellular recording at $L 5$ motoneurons (diagrams show position of stimulating and recording electrodes). $\boldsymbol{a}$, Superimposed consecutive traces demonstrating monosynaptic responses recorded in $\mathrm{L} 5$ motoneurons from IWM and polysynaptic responses in same motoneuron from contralateral LWM in noninjured cord with control treat(penicillinase + HSV-1ac vectors $+\beta$-galactosidase-secreting fibroblasts plugs, $n=28$ cells $/ 5$ rats). $c$, Hx cord, ChABC

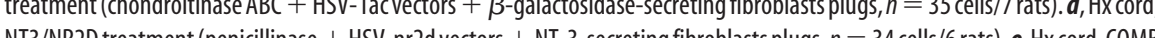
treatment (chondroitinase ABC + HSV-nr2d vectors + NT-3-secreting fibroblasts plugs $n=35$ cells $/ 6$ rats). Vertical arrows point to stimulus in all traces. Graph represents summary of results for $\boldsymbol{a}$ - $\boldsymbol{e}$ groups, respectively (mean $\pm \mathrm{SEM},{ }^{*}$ represents significant effect of full-treatment $\mathrm{Hx}$ group vs all other Hx groups, $p<0.05$ ).

et al., 2008). Following BDA injections the traced reticulospinal tract axons were visualized predominantly in the ventrolateral and ventral funiculi of the spinal cord (Fig. $6 a$ ). We quantified the sprouting of these axons by counting the number of branches from these axons in the white matter, and we determined the innervation of the spinal cord gray matter by counting the number of branches crossing from white to gray matter in five horizontal sections of lateral to ventrolateral cord. In each section the number of branches or crossings was divided by the number of labeled axons to give a sprouting ratio. These measures were taken $2 \mathrm{~mm}$ above and below the lesion on the contralateral side, and $2 \mathrm{~mm}$ above the lesion on the ipsilateral side (Fig. 6). At T8 in uninjured animals occasional branching and crossings of the gray matter boundary by reticulospinal axons were seen, and some terminal arborization was seen in lamina VIII $(n=4$, data not shown). PEN animals displayed a few processes crossing the white-gray matter boundary (Fig. 6). Counting the traced reticulospinal axons revealed that animals receiving $\mathrm{ChABC}$ alone $(n=$ 3) or in combination with NT-3 and enhanced NR2D expression 


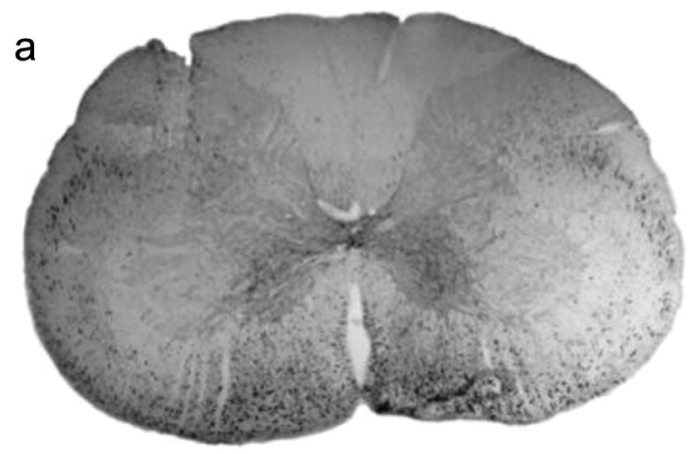

BDA

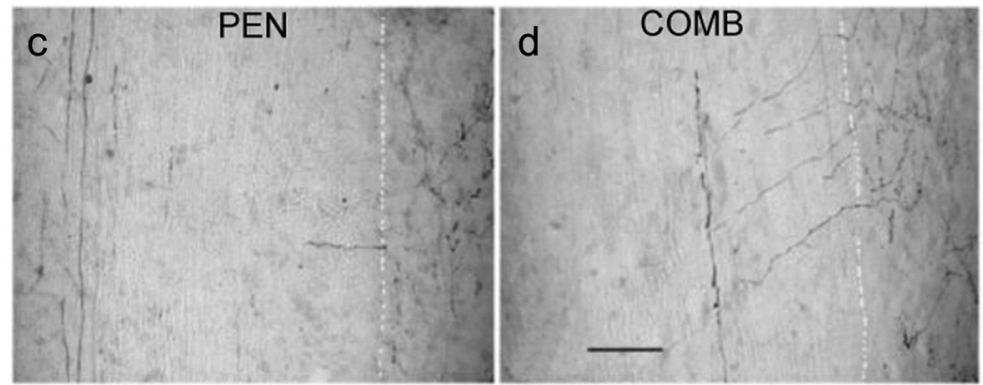

b
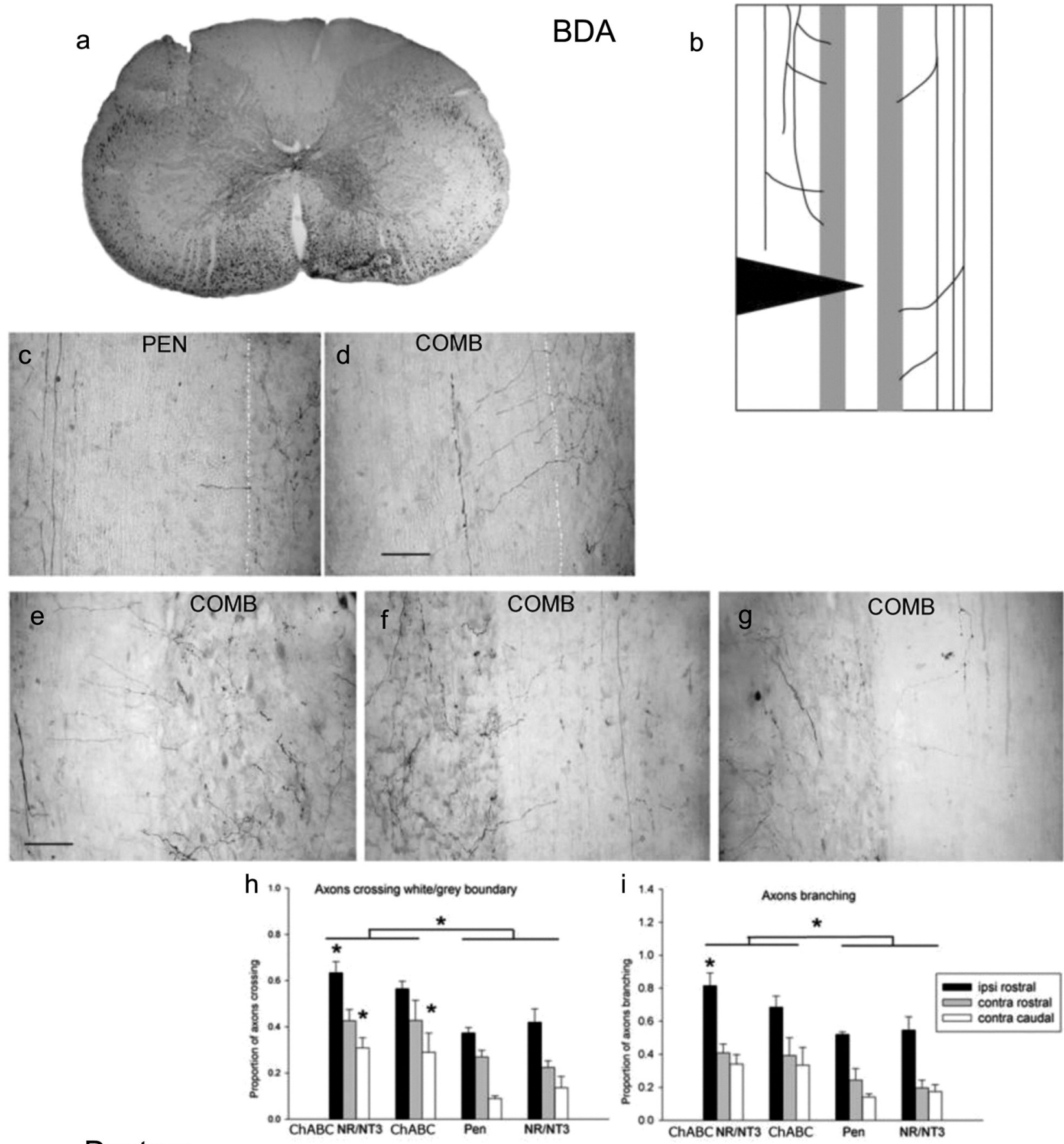

\section{Dextran}
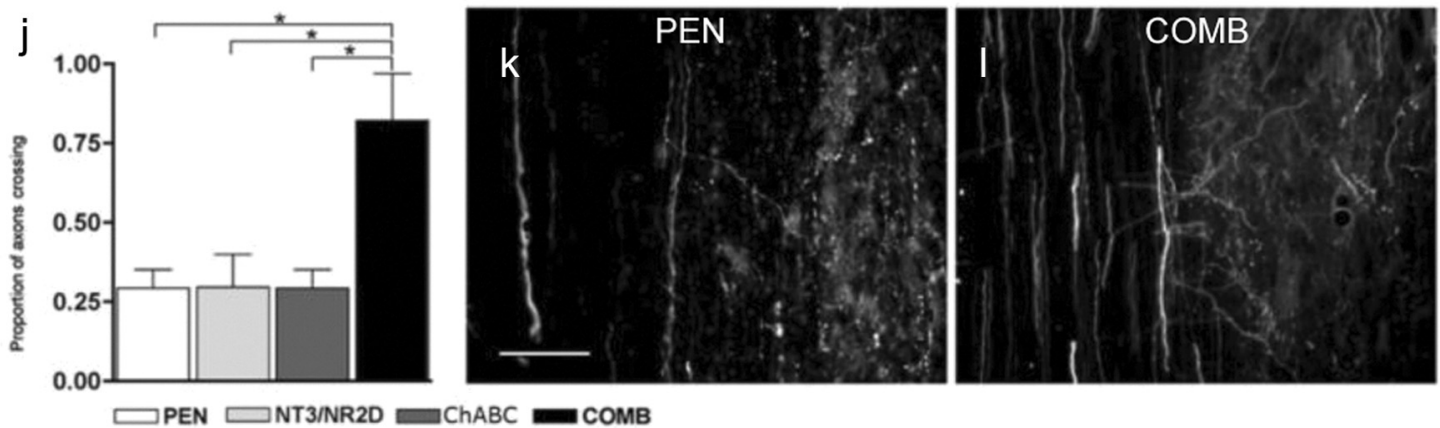

Figure 6. Axonal plasticity at the site of injury. BDA was applied bilaterally to the brainstem reticular formation. $\boldsymbol{a}$, Traced reticulospinal axons were localized mostly in the lateral and ventral funiculi. $\boldsymbol{b}$, In alternate longitudinal sections, the number of BDA-stained axons branching and the number crossing the white matter-gray matter boundary were counted (Figure legend continues.) 
$(n=5)$ displayed more sprouting and more axons crossing white-gray matter boundary than those that received PEN $(n=$ 3 ) or NT3 and enhanced NR2D expression $(n=3)$ (Fig. $6 h)$. The COMB animals exhibited more axon branching than the other groups (Fig. $6 i$ ). There were some labeled processes ipsilaterally around and below the lesion, but because tracts were labeled bilaterally it was not possible to determine whether these were sprouts from uninjured axons or regenerated process, so these neurites were not counted.

In addition, in a group of animals, fluoro-dextran tracer was injected bilaterally in the ventrolateral cord at level T6, labeling axons (rubrospinal, reticulospinal, propriospinal, ascending sensory pathways, etc.) indiscriminately in this part of the cord (see Materials and Methods). As with the axons traced from reticulospinal injections, many axons branched, and we counted the number that crossed the white-gray matter boundary. In this assay, many more axon sprouts crossing the white matter boundary were seen in COMB animals than in the other experimental groups [ $p<0.05$ vs PEN $(n=6), p<0.05$ vs NT3, and enhanced NR2D expression $(n=3), p<0.05$ vs ChABC $(n=6)$ (Fig. $6 f)]$.

\section{Discussion}

Overall our results show that the combination of ChABC, NT3 and enhanced NR2D expression is more effective in promoting sprouting of reticulospinal axons, in restoring projections from LWM (including but not limited to reticulospinal axons) to motoneurons, and in encouraging behavioral recovery than either ChABC alone or the combination of NT3 and enhanced NR2D expression.

\section{Axonal plasticity \\ The BDA tracing results show that rats treated with $\mathrm{ChABC}$ alone exhibited reticulospinal sprouting, but animals treated with all 3 agents had greater reticulospinal axon sprouting. The separate NT3/NR2D and ChABC groups did not show increased sprout- ing of LWM axons identified by injecting fluoro dextran tracer directly into white matter tracts at T6. This suggests that either reticulospinal axons were not labeled by this procedure or that they constituted such a small proportion of the total that the sprouting was not evident. More importantly, these findings in- dicate that factors determining axonal sprouting are dependent on the axon population being considered. In agreement with the findings on reticulospinal axons, a massive number of LWM ax- ons was observed crossing the white-gray matter boundary in spinal cords with the combined treatment.}

\section{$\leftarrow$}

(Figure legend continued.) at three points: rostral and caudal to the lesion (black wedge) on the contralateral side, and rostral to the lesion on the ipsilateral side. The diagram is arranged with rostral upward and the lesion on the left, as are the illustrations in $\boldsymbol{c}-\boldsymbol{g}$ and $\boldsymbol{j}-\boldsymbol{I}) . \boldsymbol{c}, \boldsymbol{d}$, Comparison of sprouting ipsilateral to the lesion in PEN (c) and COMB (d) animals. The graywhite boundary is marked with white spots. $\boldsymbol{e}-\boldsymbol{g}$, Pictures from a single COMB animal taken from ipsilateral and rostral to the lesion $(\boldsymbol{e})$, contralateral and rostral $(\boldsymbol{f})$, and contralateral and caudal ( $\boldsymbol{g}) . \boldsymbol{h}, \boldsymbol{i}$, Quantification of sprouting of axons labeled from the reticular formation, with axons crossing the gray-white boundary in $\boldsymbol{h}$, and axon branches in white matter (i). Asterisks above the bars indicate which individual bar differed from the equivalent measure in the PEN group. The grouped statistics indicate that all the bars in the chondroitinase-treated animals, pooled together, differed from the equivalent bar in the animals that did not receive chondroitinase, ${ }^{*} p<0.05 . j-I$, Fluoro-dextran tracer was injected bilaterally into the lateral funiculiat $\mathrm{T} 6$. $\boldsymbol{j}$ shows quantification of traced axons crossing the gray-white boundary. $\boldsymbol{k}, \boldsymbol{I}$, Images of PEN and COMB Fluoro-dextran traced axons crossing the white-gray matter boundary rostral and ipsilateral to the lesion. Scale bars, $200 \mu \mathrm{m} .{ }^{*} p<0.05, n=3-7$ rats per group.

\section{Electrophysiological connectivity}

The electrophysiological findings provide some insights into the projection from LWM axons, their response to injury, and how they might be affected by the treatments. Animals receiving only control treatment showed a loss of projection from above the lesion for both the ipsilateral and contralateral LWM axons. The loss of the monosynaptic projection from the ipsilateral LWM resulted from interruption of axons due to Hx. The loss of projection from the unlesioned contralateral LWM resulted from conduction blockade in axons in the LWM contralateral to $\mathrm{Hx}$ (Arvanian et al., 2009). Conduction in this contralateral pathway was partially restored after administration of $\mathrm{ChABC}$, presumably due to digestion of the CSPGs responsible for the conduction block (Hunanyan et al., 2010), but the projection to motoneurons from ipsilateral LWM through the lesion remained compromised. Animals with elevated NT3 and enhanced expression of NR2D exhibited polysynaptic responses from both LWM on both sides to L5 motoneurons on the Hx side of the cord, but these responses were very small and observed in only a few animals. The COMB animals consistently displayed enhanced projections from both contralateral and ipsilateral LWM axons to L5 motoneurons in all animals.

A significant finding was that the $\mathrm{COMB}$ treatment resulted in long latency, polysynaptic potentials from ipsilateral LWM that were considerably larger than the sum of the individual effects (ChABC; NT3 and enhanced NR2D expression). This suggested a cooperative effect, i.e., that $\mathrm{ChABC}$ facilitated the effects of NT3 and enhanced NR2D expression or vice versa. This was not the case for the projection from the contralateral LWM where the result of the combined treatment was a linear combination of the individual treatments. This suggests that the COMB cooperative effect occurred close to the injury site involving development of newly functional connections across the spinal cord since the contralateral projection might also have been expected to show cooperative effects from COMB treatment if it occurred close to the motoneurons. Since the agents were applied close to the Hx, they might have been at a high enough concentration only near the site of administration to elicit the cooperative effect locally. We cannot specify the nature of the cooperative effect at this time except to speculate that the presence of high levels of ChABC enhanced local plasticity such that the sprouts produced by treatment with NT3 enhanced expression of NR2D were more successful in making new synaptic contacts with interneurons close to the injury site (Fig. 7).

One implication of this NR2D expression-dependent mechanism is that sprouted connections onto contralateral interneurons near the injury are glutamatergic. LWM fibers make glutamatergic connections to motoneurons (Hunanyan et al., 2010,2011 ) and so when these fibers sprout, their connections are likely to be glutamatergic. It has also been shown in rodent cord that lumbar commissural interneurons make glutamatergic projections (Butt and Kiehn, 2003; Talpalar et al., 2011), offering another possible site for the action of NR2D expression-dependent mechanisms. These results also do not rule out possible strengthening of inhibitory connections which might interfere with the recovery process.

In summary, we suggest that each of the agents used played a different role in recovery of function. ChABC was required to recover from conduction block in axons contralateral to Hx; NT3 and enhanced NR2D expression in combination with ChABC was important in instituting new connections around the hemisection; finally the activity of NMDA receptors in the target neurons might have been an early step for growing axons to es- 


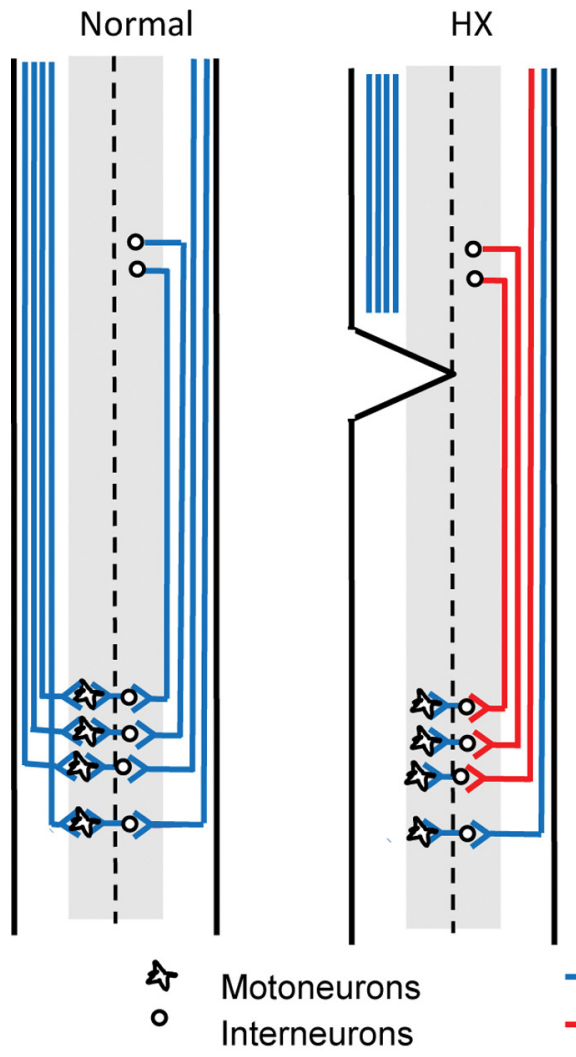

$\mathrm{HX}+\mathrm{ChABC}$

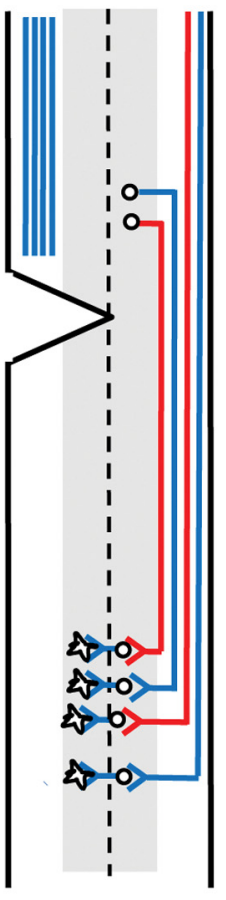

$\mathrm{HX}+\mathrm{COMB}$

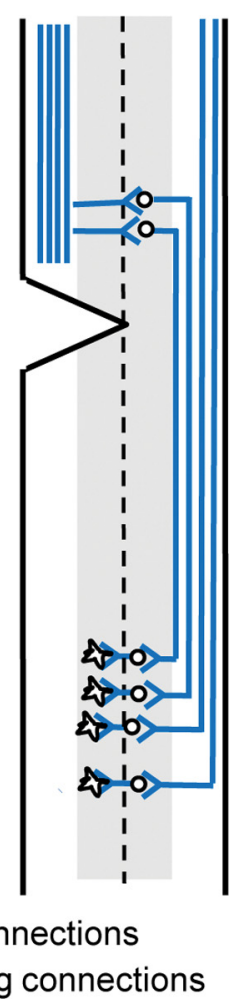

Figure 7. Diagram illustrating effect of chronic $\mathrm{Hx}$ and treatment on synaptic connections to motoneurons. Normal noninjured cord, Monosynaptic projections to motoneurons from ipsilateral and polysynaptic connections from contralateral white matter axons (long and thoracic propriospinal projections). Hx, no treatment, Monosynaptic ipsilateral projections are abolished and contralateral connections became markedly weaker. Hx, ChABC alone, Slightly stronger connections from contralateral projections, but no ipsilateral projections. Hx, COMB, Stronger projections from contralateral side and novel polysynaptic connections from ipsilateral projections around $\mathrm{Hx}$.

tablish glutamatergic synaptic contacts upon them, as in development (Ziskind-Conhaim, 1990; Kalb and Hockfield, 1992).

\section{Behavioral recovery}

During the first 2 weeks after injury all animals exhibited a similar pattern of recovery suggesting similarity in the extent of the injury. All the active treatment groups recovered better locomotion and hindlimb function than PEN animals. Overall, the COMB group displayed a trend for better recovery than the single treatment groups, but this was not statistically significant in individual tests. It is probable that the better connectivity to the lumbar spinal cord achieved following the combinatorial treatment allowed the animals to develop better postural stability, and consequently to make longer forelimb steps. However, hindlimb function remained severely impaired with no significant differences between groups in a task requiring walking across a ladder with unevenly spaced rungs.

We tested hindlimb sensory function using von Frey hair and hotplate tests. All the animals showed increased mechanical sensitivity from both limbs despite the asymmetry of the spinal lesion suggesting that considerable reorganization must have taken place in the spinal cord and/or brain. Given the substantial increase in mechanical sensitivity, it is striking that the sensitivity to noxious heat was virtually unaffected by the Hx. Also surprising was the lack of effect of the therapies, particularly the COMB therapy, which had substantial effects on the cord in both the anatomical and electrophysiological evaluations.

\section{Experimental therapies to promote spinal cord repair}

We have tested a combination of three interventions that have been shown to influence anatomical sprouting, synaptic plasticity and recovery in animal models of spinal cord injury. Delivery of NT3, by single injection (Schnell et al., 1994), or grafted fibroblasts genetically modified to secrete NT3 at the site of injury (Grill et al., 1997), or viruses carrying the NT3 gene (Taylor et al., 2006) has been shown to enhance sprouting of axons in gray matter and the strength of synapses on motoneurons (Mendell et al., 2001; Arvanian et al., 2003).

In neonatal rats NT3 administration strengthens peripheral sensory and descending LWM synapses onto lumbar motoneurons (Arvanian et al., 2003). Initiation of this neurotrophininduced plasticity may require functional NMDA receptors in motoneurons and ceases shortly after birth, at which time NR2D expression is downregulated at these synapses (Arvanian et al., 2004). In later postnatal animals the initial plasticity in response to NT3 can be restored if $\mathrm{Mg}^{2+}$ is depleted (Arvanian and Mendell, 2001) or by injecting viruses expressing the NR2D gene (Arvanian et al., 2004). The plasticity induced by combining NT3 and elevating NR2D expression strengthens synaptic transmission and sprouting in neonatal rats subjected to contusion or staggered double hemisection (Arvanian et al., 2006). However, in adult rats with chronic Hx this action of NT3 and elevated NR2D expression is weak (Fig. $5 d$ ).

Glycosaminoglycan chains of CSPGs shown here to be upregulated in this hemisection model of spinal cord injury are well known to be digested by ChABC using a protocol identical to that used here (García-Alías et al., 2008, 2009; Hunanyan et al., 2010). This achieves several beneficial modifications of the injured spinal cord. First, it creates an environment permissive for axonal growth and sprouting in the injured spinal cord by rendering the CSPGs in the glial scar less inhibitory, promoting regrowth of 
corticospinal (Bradbury et al., 2002; García-Alías et al., 2009), serotonergic (Fouad et al., 2005; Barritt et al., 2006), reticulospinal (Vavrek et al., 2007) and ascending dorsal column axons (Bradbury et al., 2002). Second, it reverses the conduction block observed in intact axons across from the Hx (Hunanyan et al., 2010). Finally, it digests CSPGs within perineuronal nets. These nets, which appear in the rodent spinal cord around postnatal day 12 , are one factor that turns off plasticity at the end of the critical period (Galtrey et al., 2008; Carulli et al., 2010). It is interesting that the combination of NT3 treatment and enhancing NR2D expression loses the ability to promote plasticity in the connections to motoneurons in the second postnatal week, which corresponds to the first appearance of perineuronal nets. In our experiments digestion of the perineuronal nets in adult animals restored the ability of NT3 treatment and elevated NR2D expression to affect connectivity after spinal cord injury.

\section{Conclusion}

These findings show the additive effects of combining chondroitinase ABC, NT3 and enhancing NR2D expression in promoting the formation or strengthening of spinal circuits and modest functional recovery. In addition, the results indicate the feasibility of combination treatments to promote spinal cord repair.

\section{References}

Afshari FT, Kappagantula S, Fawcett JW (2009) Extrinsic and intrinsic factors controlling axonal regeneration after spinal cord injury. Expert Rev Mol Med 11:e37.

Alilain WJ, Horn KP, Hu H, Dick TE, Silver J (2011) Functional regeneration of respiratory pathways after spinal cord injury. Nature 475:196-200.

Alstermark B, Ogawa J, Isa T (2004) Lack of monosynaptic corticomotoneuronal EPSPs in rats: disynaptic EPSPs mediated via reticulospinal neurons and polysynaptic EPSPs via segmental interneurons. J Neurophysiol 91:1832-1839.

Arvanian VL, Mendell LM (2001) Removal of NMDA receptor $\mathrm{Mg}(2+$ ) block extends the action of NT- 3 on synaptic transmission in neonatal rat motoneurons. J Neurophysiol 86:123-129.

Arvanian VL, Horner PJ, Gage FH, Mendell LM (2003) Chronic neurotrophin-3 strengthens synaptic connections to motoneurons in the neonatal rat. J Neurosci 23:8706-8712.

Arvanian VL, Bowers WJ, Petruska JC, Motin V, Manuzon H, Narrow WC, Federoff HJ, Mendell LM (2004) Viral delivery of NR2D subunits reduces $\mathrm{Mg}^{2+}$ block of NMDA receptor and restores NT-3-induced potentiation of AMPA-kainate responses in maturing rat motoneurons. J Neurophysiol 92:2394-2404.

Arvanian VL, Bowers WJ, Anderson A, Horner PJ, Federoff HJ, Mendell LM (2006) Combined delivery of neurotrophin-3 and NMDA receptors 2D subunit strengthens synaptic transmission in contused and staggered double hemisected spinal cord of neonatal rat. Exp Neurol 197:347-352.

Arvanian VL, Schnell L, Lou L, Golshani R, Hunanyan A, Ghosh A, Pearse DD, Robinson JK, Schwab ME, Fawcett JW, Mendell LM (2009) Chronic spinal hemisection in rats induces a progressive decline in transmission in uninjured fibers to motoneurons. Exp Neurol 216:471-480.

Ballermann M, Fouad K (2006) Spontaneous locomotor recovery in spinal cord injured rats is accompanied by anatomical plasticity of reticulospinal fibers. Eur J Neurosci 23:1988-1996.

Barritt AW, Davies M, Marchand F, Hartley R, Grist J, Yip P, McMahon SB, Bradbury EJ (2006) Chondroitinase ABC promotes sprouting of intact and injured spinal systems after spinal cord injury. J Neurosci 26:10856-10867.

Basso DM, Beattie MS, Bresnahan JC (1995) A sensitive and reliable locomotor rating scale for open field testing in rats. J Neurotrauma 12:1-21.

Bowers WJ, Howard DF, Federoff HJ (2000) Discordance between expression and genome transfer titering of HSV amplicon vectors: recommendation for standardized enumeration. Mol Ther 1:294-299.

Bowers WJ, Howard DF, Brooks AI, Halterman MW, Federoff HJ (2001) Expression of vhs and VP16 during HSV-1 helper virus-free amplicon packaging enhances titers. Gene Ther 8:111-120.
Bradbury EJ, Moon LD, Popat RJ, King VR, Bennett GS, Patel PN, Fawcett JW, McMahon SB (2002) Chondroitinase ABC promotes functional recovery after spinal cord injury. Nature 416:636-640.

Busch SA, Silver J (2007) The role of extracellular matrix in CNS regeneration. Curr Opin Neurobiol 17:120-127.

Butt SJ, Kiehn O (2003) Functional identification of interneurons responsible for left-right coordination of hindlimbs in mammals. Neuron 38:953-963.

Carulli D, Pizzorusso T, Kwok JC, Putignano E, Poli A, Forostyak S, Andrews MR, Deepa SS, Glant TT, Fawcett JW (2010) Animals lacking link protein have attenuated perineuronal nets and persistent plasticity. Brain 133:2331-2347

Davies JE, Tang X, Bournat JC, Davies SJ (2006) Decorin promotes plasminogen/plasmin expression within acute spinal cord injuries and by adult microglia in vitro. J Neurotrauma 23:397-408.

Fidler PS, Schuette K, Asher RA, Dobbertin A, Thornton SR, Calle-Patino Y, Muir E, Levine JM, Geller HM, Rogers JH, Faissner A, Fawcett JW (1999) Comparing astrocytic cell lines that are inhibitory or permissive for axon growth: the major axon-inhibitory proteoglycan is NG2. J Neurosci 19:8778-8788

Fouad K, Schnell L, Bunge MB, Schwab ME, Liebscher T, Pearse DD (2005) Combining Schwann cell bridges and olfactory-ensheathing glia grafts with chondroitinase promotes locomotor recovery after complete transection of the spinal cord. J Neurosci 25:1169-1178.

Galtrey CM, Asher RA, Nothias F, Fawcett JW (2007) Promoting plasticity in the spinal cord with chondroitinase improves functional recovery after peripheral nerve repair. Brain 130:926-939.

Galtrey CM, Kwok JC, Carulli D, Rhodes KE, Fawcett JW (2008) Distribution and synthesis of extracellular matrix proteoglycans, hyaluronan, link proteins and tenascin- $\mathrm{R}$ in the rat spinal cord. Eur J Neurosci 27:1373-1390.

García-Alías G, Arvanian VL, Schnell L, Horner PJ, Bowers WJ, Federoff HJ, Levine J, Fawcett JW, Mendell LM (2006) Recovery of hindlimb motor function after a lateral spinal cord hemisection in adult rat is enhanced by combined administration of NT-3, NMDA-2D subunits and chondroitinase-ABC. Soc Neurosci Abstr 32:646.21.

García-Alías G, Lin R, Akrimi SF, Story D, Bradbury EJ, Fawcett JW (2008) Therapeutic time window for the application of chondroitinase $\mathrm{ABC}$ after spinal cord injury. Exp Neurol 210:331-338.

García-Alías G, Barkhuysen S, Buckle M, Fawcett JW (2009) Chondroitinase $A B C$ treatment opens a window of opportunity for task-specific rehabilitation. Nat Neurosci 12:1145-1151.

Girgis J, Merrett D, Kirkland S, Metz GA, Verge V, Fouad K (2007) Reaching training in rats with spinal cord injury promotes plasticity and task specific recovery. Brain 130:2993-3003.

Grill R, Murai K, Blesch A, Gage FH, Tuszynski MH (1997) Cellular delivery of neurotrophin-3 promotes corticospinal axonal growth and partial functional recovery after spinal cord injury. J Neurosci 17:5560-5572.

Hains BC, Johnson KM, McAdoo DJ, Eaton MJ, Hulsebosch CE (2001) Engraftment of serotonergic precursors enhances locomotor function and attenuates chronic central pain behavior following spinal hemisection injury in the rat. Exp Neurol 171:361-378.

Hargreaves K, Dubner R, Brown F, Flores C, Joris J (1988) A new and sensitive method for measuring thermal nociception in cutaneous hyperalgesia. Pain 32:77-88.

Hill CE, Beattie MS, Bresnahan JC (2001) Degeneration and sprouting of identified descending supraspinal axons after contusive spinal cord injury in the rat. Exp Neurol 171:153-169.

Houle JD, Tom VJ, Mayes D, Wagoner G, Phillips N, Silver J (2006) Combining an autologous peripheral nervous system "bridge" and matrix modification by chondroitinase allows robust, functional regeneration beyond a hemisection lesion of the adult rat spinal cord. J Neurosci 26:7405-7415.

Hunanyan AS, García-Alías G, Alessi V, Levine JM, Fawcett JW, Mendell LM, Arvanian VL (2010) Role of chondroitin sulfate proteoglycans in axonal conduction in Mammalian spinal cord. J Neurosci 30:7761-7769.

Hunanyan AS, Alessi V, Patel S, Pearse DD, Matthews G, Arvanian VL (2011) Alterations of action potentials and the localization of Nav1.6 sodium channels in spared axons after hemisection injury of the spinal cord in adult rats. J Neurophysiol 105:1033-1044.

Kalb RG, Hockfield S (1992) Activity-dependent development of spinal cord motor neurons. Brain Res Brain Res Rev 17:283-289. 
Karimi-Abdolrezaee S, Eftekharpour E, Wang J, Schut D, Fehlings MG (2010) Synergistic effects of transplanted adult neural stem/progenitor cells, chondroitinase, and growth factors promote functional repair and plasticity of the chronically injured spinal cord. J Neurosci 30:1657-1676.

Kawaja MD, Gage FH (1992) Morphological and neurochemical features of cultured primary skin fibroblasts of Fischer 344 rats following striatal implantation. J Comp Neurol 317:102-116.

Kim BG, Dai HN, Lynskey JV, McAtee M, Bregman BS (2006) Degradation of chondroitin sulfate proteoglycans potentiates transplant-mediated axonal remodeling and functional recovery after spinal cord injury in adult rats. J Comp Neurol 497:182-198.

Kwok JC, Afshari F, García-Alías G, Fawcett JW (2008) Proteoglycans in the central nervous system: plasticity, regeneration and their stimulation with chondroitinase ABC. Restor Neurol Neurosci 26:131-145.

Liebscher T, Schnell L, Schnell D, Scholl J, Schneider R, Gullo M, Fouad K, Mir A, Rausch M, Kindler D, Hamers FP, Schwab ME (2005) Nogo-A antibody improves regeneration and locomotion of spinal cord-injured rats. Ann Neurol 58:706-719.

Lilley CE, Branston RH, Coffin RS (2001) Herpes simplex virus vectors for the nervous system. Curr Gene Ther 1:339-358.

Lou L, García-Alías G, Mendell LM, Fawcett JW, Arvanian VL (2007) Transmission through white matter contralateral to thoracic hemisection declines beginning several days after injury in parallel with enhanced expression of CSPGs in the vicinity of the lesion. Soc Neurosci Abstr 33:801.1.

Lu P, Tuszynski MH (2008) Growth factors and combinatorial therapies for CNS regeneration. Exp Neurol 209:313-320.

Maier IC, Schwab ME (2006) Sprouting, regeneration and circuit formation in the injured spinal cord: factors and activity. Philos Trans R Soc Lond B Biol Sci 361:1611-1634.

Massey JM, Amps J, Viapiano MS, Matthews RT, Wagoner MR, Whitaker CM, Alilain W, Yonkof AL, Khalyfa A, Cooper NG, Silver J, Onifer SM (2008) Increased chondroitin sulfate proteoglycan expression in denervated brainstem targets following spinal cord injury creates a barrier to axonal regeneration overcome by chondroitinase $\mathrm{ABC}$ and neurotrophin-3. Exp Neurol 209:426-445.

McTigue DM, Horner PJ, Stokes BT, Gage FH (1998) Neurotrophin-3 and brain-derived neurotrophic factor induce oligodendrocyte proliferation and myelination of regenerating axons in the contused adult rat spinal cord. J Neurosci 18:5354-5365.

Mendell LM, Munson JB, Arvanian VL (2001) Neurotrophins and synaptic plasticity in the mammalian spinal cord. J Physiol 533:91-97.

Metz GA, Schwab ME (2004) Behavioral characterization in a comprehen- sive mouse test battery reveals motor and sensory impairments in growthassociated protein-43 null mutant mice. Neuroscience 129:563-574.

Paxinos G, Watson C (1998) The rat brain in stereotaxic coordinates. San Diego: Academic.

Reed WR, Shum-Siu A, Magnuson DS (2008) Reticulospinal pathways in the ventrolateral funiculus with terminations in the cervical and lumbar enlargements of the adult rat spinal cord. Neuroscience 151:505-517.

Schnell L, Schneider R, Kolbeck R, Barde YA, Schwab ME (1994) Neurotrophin-3 enhances sprouting of corticospinal tract during development and after adult spinal cord lesion. Nature 367:170-173.

Schucht P, Raineteau O, Schwab ME, Fouad K (2002) Anatomical correlates of locomotor recovery following dorsal and ventral lesions of the rat spinal cord. Exp Neurol 176:143-153.

Shearer MC, Niclou SP, Brown D, Asher RA, Holtmaat AJ, Levine JM, Verhaagen J, Fawcett JW (2003) The astrocyte/meningeal cell interface is a barrier to neurite outgrowth which can be overcome by manipulation of inhibitory molecules or axonal signalling pathways. Mol Cell Neurosci 24:913-925.

Silver J, Miller JH (2004) Regeneration beyond the glial scar. Nat Rev Neurosci 5:146-156.

Snow DM, Lemmon V, Carrino DA, Caplan AI, Silver J (1990) Sulfated proteoglycans in astroglial barriers inhibit neurite outgrowth in vitro. Exp Neurol 109:111-130.

Talpalar AE, Endo T, Löw P, Borgius L, Hägglund M, Dougherty KJ, Ryge J, Hnasko TS, Kiehn O (2011) Identification of minimal neuronal networks involved in flexor-extensor alternation in the Mammalian spinal cord. Neuron 71:1071-1084.

Taylor L, Jones L, Tuszynski MH, Blesch A (2006) Neurotrophin-3 gradients established by lentiviral gene delivery promote short-distance axonal bridging beyond cellular grafts in the injured spinal cord. J Neurosci 26:9713-9721.

Vavrek R, Pearse DD, Fouad K (2007) Neuronal populations capable of regeneration following a combined treatment in rats with spinal cord transection. J Neurotrauma 24:1667-1673.

You SW, Chen BY, Liu HL, Lang B, Xia JL, Jiao XY, Ju G (2003) Spontaneous recovery of locomotion induced by remaining fibers after spinal cord transection in adult rats. Restor Neurol Neurosci 21:39-45.

Zemanick MC, Strick PL, Dix RD (1991) Direction of transneuronal transport of herpes simplex virus 1 in the primate motor system is straindependent. Proc Natl Acad Sci U S A 88:8048-8051.

Ziskind-Conhaim L (1990) NMDA receptors mediate poly- and monosynaptic potentials in motoneurons of rat embryos. J Neurosci 10:125-135. 